\title{
Silencing of CerS6 increases the invasion and glycolysis of melanoma WM35, WM451 and SK28 cell lines via increased GLUT1-induced downregulation of WNT5A
}

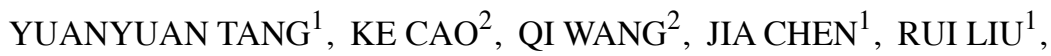 \\ SHAOHUA WANG ${ }^{1}$, JIANDA ZHOU ${ }^{1}$ and HUIQING XIE ${ }^{3}$ \\ Departments of ${ }^{1}$ Plastic Surgery, ${ }^{2}$ Oncology and ${ }^{3}$ Rehabilitation, The Third Xiangya Hospital \\ of Central South University, Changsha, Hunan 410013, P.R. China
}

Received November 10, 2015; Accepted December 17, 2015

DOI: $10.3892 /$ or.2016.4646

\begin{abstract}
Ceramide synthases (CerSs) have been shown to regulate numerous aspects of cancer development. CerS6 has been suggested to be involved in cancer etiology. However, little is known concerning the exact effect of CerS6 on the malignant behavior of melanoma, including glycolysis, proliferation and invasion. In the present study, we found that the expression of CerS6 was low in the melanoma cell lines, including WM35, WM451 and SK-28, and the expression level was related to the malignanct behavior of the melanoma cell lines. We constructed overexpression and silencing models of CerS6 in three melanoma cell lines and found that silencing of CerS6 promoted the ability of proliferation and invasion in the melanoma cell lines. Additionally, downregulation of CerS6 upregulated the activity of glycolysis-related enzyme, and enhanced the expression of glycolysis-related genes, including GLUT1 and MCT1. Furthermore, we identified the genes whose expression levels were changed after silencing of CerS6 by gene microarray. The expression of glycolysisrelated gene SLC2A1 (also known as GLUT1) was found to be upregulated, while notably WNT5A was downregulated. The altered expression of GLUT1 and WNT5A was verified by qPCR and western blotting. Furthermore, silencing of GLUT1 in the melanoma cells resulted in the increased expression of WNT5A and the decreased ability of invasion and proliferation in the melanoma cells. Collectively, silencing of CerS6
\end{abstract}

Correspondence to: Dr Jianda Zhou, Department of Plastic Surgery, The Third Xiangya Hospital of Central South University, 138 Tongzipo Road, Changsha, Hunan 410013, P.R. China E-mail: zhoujianda_csu@163.com

Dr Huiqing Xie, Department of Rehabilitation, The Third Xiangya Hospital of Central South University, The Third Xiangya Hospital of Central South University, 138 Tongzipo Road, Changsha, Hunan 410013, P.R. China

E-mail: 975979752@qq.com

Key words: melanoma, glycolysis, ceramide, GLUT1, CerS6, WNT5A induced the increased expression of GLUT1, which downregulated the expression of WNT5A and enhanced the invasion and proliferation of melanoma cells. Thus, CerS6 may provide a novel therapeutic target for melanoma treatment.

\section{Introduction}

Melanoma, a malignancy that arises from melanocytes, accounts for $\sim 10 \%$ of all skin tumors (1). It is the most aggressive skin cancer and is characterized by abnormal proliferation of melanocytes (2). Due to its high metastatic potential and strong resistance to radiation, immunotherapy and chemotherapy, the search for novel anti-melanoma therapies is urgent (2). Currently, it is widely accepted that metabolic changes are one of the hallmarks of cancer (3). Accordingly in recent years, cancer therapeutics are focused on two metabolic fields: glycolytic metabolism and bioactive sphingolipid synthesis (4).

Increased glycolysis in tumor cells compared to normal tissues is observed in most types of cancers and supports the increased energy and biosynthetic demands of tumor cells (5). This is in accordance with the Warburg hypothesis which posits that aerobic glycolysis is a major source of energy in malignant cells (6). Therefore, the glycolytic metabolism is an important target for regulating tumor progression.

Ceramide, a backbone of the sphingolipid family, is not only a component of the membrane structure, but also is an essential mediator of cellular functions, such as growth, differentiation and apoptosis (7-10). Disturbances in ceramide synthesis and signaling have been implicated in many types of cancers $(8,11,12)$. In human melanoma cell lines, resistance to stress-induced apoptosis has been associated with low ceramide levels (13). Additionally, an increased intracellular level of ceramide was found to inhibit the cell proliferation and promote the apoptosis of tumor cells (9). Thus, strategies targeting ceramide synthesis in cancer cells have been developed as novel approaches for anticancer chemotherapy $(14,15)$.

Ceramide can be produced via two distinct pathways, one of which is de novo by a family of genes known as ceramide synthases (CerSs), which consists of six members, CerS1 to 
CerS6 $(16,17)$. In addition to regulating sphingolipid synthesis, CerS activity has also been shown to regulate numerous functions of cell biology, including cell growth, apoptosis, autophagy and particularly cancer development (18-21). As an important member of the CerS family, various studies have suggested that CerS6 is involved in cancer etiology. For example, knockdown of CerS6 resulted in a specific decrease in intracellular C16-ceramide, protected colon adenocarcinoma cells against TRAIL-mediated apoptosis and interfered with translocation of active caspase-3 into the nucleus. In contrast, increased CerS6 expression sensitized the cells to TRAIL (22). Senkal et al demonstrated the anti-apoptotic role of CerS6 in head and neck squamous cell carcinoma (HNSCC) (23). Downregulation of CerS6 in HNSCC resulted in the induction of ER stress, while overexpression of CerS6 increased HNSCC tumor development and growth (24). However, little is known concerning the exact effect of CerS6 on the malignant behavior of melanoma.

In the present study, we found that the expression of CerS6 in three melanoma cell lines, including WM35, WM451 and SK-MEL-28 (SK-28) was low. We further overexpressed and knocked down CerS6 in the three melanoma cell lines. The effect of CerS6 on the invasion, proliferation, and glycolysis in melanoma cells was evaluated, respectively. Furthermore, we detected the genes that had altered expression after CerS6 silencing by human gene chip, and found that the expression levels of GLUT1 and WNT5A in the CerS6-silenced cells were greatly altered, which was confirmed by qPCR. Moreover, the role of human glucose transporter GLUT1 in regulating the WNT5A expression and the invasion and proliferation of the melanoma cells was then analyzed. The present study may provide a novel therapeutic target for clinical melanoma treatment.

\section{Materials and methods}

Cell culture. Human melanoma cell lines, including WM35 (25), WM451 (25) and SK28 (26), HaCaT (27) and Hm (25) cells were purchased from the Chinese Academy of Sciences (Shanghai, China). Cells were cultured in Dulbecco's modified Eagle's medium (DMEM; HyClone, UK) with $10 \%$ fetal bovine serum (FBS; Gibco, USA) in an $37^{\circ} \mathrm{C}$ atmosphere of $5 \% \mathrm{CO}_{2}$.

Real-time RT-PCR assay. Total RNA of the cells was extracted using TriPure reagent (Roche, Shanghai, China). A reverse transcription kit (Fermentas, USA) was used to convert RNA into cDNA, according to the manufacturer's instructions. For mRNA detection, real-time PCR was conducted using a qPCR detection kit on ABI 7500 thermocycler (both from Life Technologies). $\beta$-actin was used as an internal reference. The PCR reaction conditions were $95^{\circ} \mathrm{C}$ for $5 \mathrm{~min}$, followed by 40 cycles of $95^{\circ} \mathrm{C}$ for $15 \mathrm{sec}$ and $60^{\circ} \mathrm{C}$ for $30 \mathrm{sec}$. The primer sequences were as follows: human MCT1 (F, CCA ACC CTA AGA TTA CTT CAC A and R, TCT GCC ATG ATA GCA ACA A); human CerS6 (F, TGG TGC GGC TCA TCT TC and R, CAT CCC AGT CCA GTT GCT T); human WNT5A (F, ACC GCT TTG CCA AGG AGT TCG and R, GCC TCG TTG TTG TGC AGG TTC AT); human GLUT1 (F, GCA TCG TCG TCG GCA TCC T and R, GGT TCT CCT CGT TGC GGT TG).
ELISA. The activity of glycolysis-related enzymes was detected using an ELISA kit (Huamei, Wuhan, China) in accordance with the manufacturer's instructions.

Transfection. Melanoma cells were cultured as described above before transfection. To knock down the endogenous expression of CerS6, a CerS6 inhibitor (the recombinant CerS6-shRNA) was used, while to upregulate the expression of CerS6, a recombinant plasmid with overexpression of CerS6 (both from GeneChem Co., Ltd., Shanghai, China) was used.

GLUT1-siRNA was used to regulate the expression of GLUT1. Transfection was performed using Lipofectamine ${ }^{\mathrm{TM}} 2000$ (Invitrogen, USA) according to the manufacturer's protocol. Briefly, the diluted Lipofectamine 2000 was added into the diluted plasmid. After incubation at room temperature for $20 \mathrm{~min}$, the above mixture was added into the cell suspension, which was then incubated at $37^{\circ} \mathrm{C}$, in $5 \% \mathrm{CO}_{2}$ for $6 \mathrm{~h}$. After that, the transfection mixture was replaced with DMEM with $10 \%$ FBS. Cells were then cultured for $48 \mathrm{~h}$ before the following assays.

Western blotting. Cells were lysed in cold RIPA buffer (Life Technologies). The protein concentration was determined using the BCA protein assay kit (Pierce Chemical, Rockford, IL, USA). Protein was separated with $10 \%$ SDS-PAGE, transferred to a polyvinylidene difluoride (PVDF) membrane (Life Technologies), and then blocked in 5\% non-fat dried milk (Yili, Beijing, China) in Dulbecco's phosphate-buffered saline (DPBS) (Life Technologies) for $4 \mathrm{~h}$. The PVDF membrane was then incubated with a monoclonal antibody (1:100) or human anti- $\beta$-actin monoclonal antibody (1:100) (both from Abcam, Cambridge, MA, USA) as an internal reference for $3 \mathrm{~h}$ at room temperature, and then washed with DPBS for $10 \mathrm{~min}$. After that, the PVDF membrane was incubated with a secondary antibody (1:20,000; Abcam) for $1 \mathrm{~h}$ at room temperature. After being washed with DPBS for $15 \mathrm{~min}$, the immune complexes on the PVDF membrane were detected using the ECL western blotting kit (Pierce Chemical). Image-Pro Plus software 6.0 (Media Cybernetics, Inc., Rockville, MD, USA) was used to analyze the relative protein expression, represented as the density ratio vs. $\beta$-actin.

GeneChip experiments. Total RNA was isolated from the human melanoma cell lines with TRIzol reagent (Invitrogen). A reverse transcription kit (Fermentas) was used to convert RNA into cDNA according to the manufacturer's instructions. The gene expression detection was performed by PrimeView ${ }^{\mathrm{TM}}$ Human Gene Expression Array (Affymetrix, Inc., Santa Clara, CA, USA).

Detection of cell proliferation. Cell proliferation was detected by MTT assay. Cells (10,000/group) were plated into 96-well plates and cultured at $37^{\circ} \mathrm{C}$ in $5 \% \mathrm{CO}_{2}$ for 24,48 and $72 \mathrm{~h}$. MTT $(20 \mu \mathrm{l})(5 \mathrm{mg} / \mathrm{ml}$; Life Technologies) was added into the plates and incubated at $37^{\circ} \mathrm{C}$ for $4 \mathrm{~h}$, followed by addition of $150 \mu \mathrm{l}$ of dimethyl sulfoxide (DMSO). After incubation at room temperature for $10 \mathrm{~min}$, the optical density (OD) at $570 \mathrm{~nm}$ was detected to evaluate the formazan production using a Multiskan FC enzyme immunoassay analyzer (Thermo Fisher Scientific). 
A

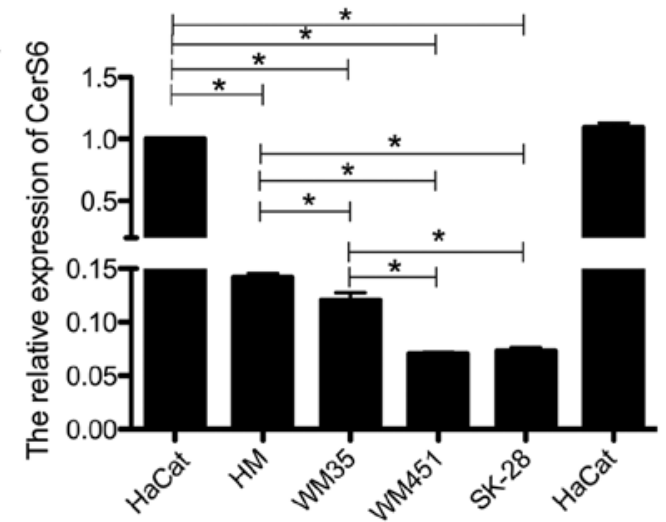

B

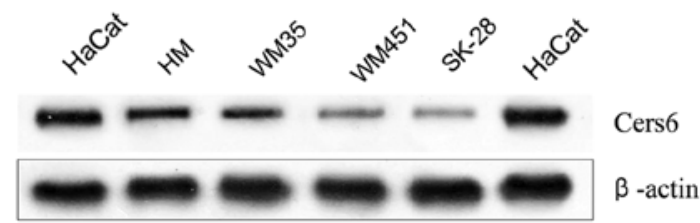

Figure 1. Expression of CerS6 is downregulated in melanoma cell lines compared with the level in the HaCaT cells. (A) The relative mRNA expression of CerS6 in different cell lines, including HaCaT, HM, WM35, WM451 and SK28. (B) Representative blot of CerS6 protein expression as measured by western blotting. Data are shown as mean $\pm \mathrm{SD}, \mathrm{n}=3$. ${ }^{*} \mathrm{P}<0.05$.

Colony formation assay. Cells (200/well) were cultured in a 6 -well plate. Plates were incubated at $37^{\circ} \mathrm{C}$ for 2 weeks. The cells were then gently washed, followed by staining with crystal violet. Viable colonies containing at least 50 cells were counted. Colony formation rate was determined as: the number of colonies/the seeded cell numbers.

Cell migration assay. Transwell chambers (BD, USA) were used for migration analysis. A total of $5 \times 10^{5}$ cells $/ \mathrm{ml}$ suspended cells was prepared in serum-free media, and $300 \mu \mathrm{l}$ of the cell suspension was added into the upper chamber. Meanwhile, $500 \mu 1$ DMEM with $10 \%$ FBS was added into the lower chamber. After that, the cells were incubated at $37^{\circ} \mathrm{C}$ for $48 \mathrm{~h}$. A cotton-tipped swab was used to carefully wipe off the cells that did not migrate through the pores. The filters were then fixed in $90 \%$ alcohol and stained by $0.1 \%$ crystal violet. The filters were then observed under an inverted microscope (Olympus, Japan).

Statistical analysis. Data are expressed as the mean \pm SD. Data analysis was performed using GraphPad Prism 6.0 software (GraphPad Software, San Diego, CA, USA). Student's t-test or one-way ANOVA was used to analyze the significance of differences among groups depending on the experimental conditions. Statistical significance was considered at $\mathrm{P}<0.05$.

\section{Results}

Expression level of CerS6 is low in the melanoma cell lines. We detected the expression of CerS6 in human immortalized epidermal cell line HaCaT, human normal melanophore HM, and three melanoma cell lines with different grades of malignancy, including WM35, WM451 and SK-28. The mRNA expression of CerS6 in the three melanoma cell lines was significantly reduced when compared with that in the HaCaT cells $(\mathrm{P}<0.05$; Fig. 1A). Additionally, CerS6 was significantly highly expressed in the WM35 cell line when compared to its level in the WM451 $(\mathrm{P}<0.05)$ and SK-28 cells $(\mathrm{P}<0.05)$. This was further verified by western blotting at the protein level of CerS6 (Fig. 1B). This indicated that the expression level of CerS6 may be closely related to the malignancy of melanoma cells.
Decreased CerS6 expression promotes the ability of invasion and proliferation in melanoma cell lines. In order to detect the effects of CerS6 on melanoma, we carried out transfection in the WM35, WM451 and SK-28 cell lines. The mRNA expression of CerS6 was significantly upregulated after transfection with the CerS6-overexpressing plasmid $(\mathrm{P}<0.01)$, and was decreased when shRNA was transfected into the cell lines $(\mathrm{P}<0.05)$ (Fig. 2A). In the colony formation assay, the CerS6-overexpressing cell lines formed fewer colonies (WM35, $\mathrm{P}<0.01$; WM451, $\mathrm{P}<0.05$; SK-28, $\mathrm{P}<0.05)$, and the number of colonies was increased after CerS6 silencing (WM35, $\mathrm{P}<0.05$; WM451, $\mathrm{P}<0.05$; SK-28, P<0.01) (Fig. 2B and C). Additionally, cell proliferation was measured by MTT assay in the WM35, WM451 and SK-28 cells. CerS6-overexpressing cells showed significantly reduced cell viability, which was increased in the CerS6-silenced WM35 $(\mathrm{P}<0.05)$ and WM451 cells $(\mathrm{P}<0.05)$, while no changes were observed in the SK-28 cells (Fig. 2D). Furthermore, we detected the invasive ability of the cell lines by Transwell assay (Fig. 2E). Overexpression of CerS6 reduced the invasion capability of the melanoma cells. In contrast, the silencing of CerS6 expression led to a significantly increased number of cells migrating through the Transwell membrane. These collectively indicated that silencing of CerS6 promoted the invasion and proliferation ability of the melanoma cell lines.

Decreased CerS6 expression upregulates glycolysis in the melanoma cell lines. To analyze the effect of CerS6 on the glycolysis of melanoma cell lines, we firstly detected the activity of several key enzymes involved in glycolysis (HK, M2-PK, PFK1 and LDHA). As depicted in Fig. 3A, overexpression of CerS6 significantly downregulated the enzyme activity of HK, M2-PK, PFK1 and LDHA in the WM35 cells (HK, $\mathrm{P}<0.01$; M2-PK, $\mathrm{P}<0.05$; PFK1, $\mathrm{P}<0.01$; LDHA, $\mathrm{P}<0.05)$, in the WM451 cells (HK, $\mathrm{P}<0.01$; M2-PK, $\mathrm{P}<0.01$; PFK1, $\mathrm{P}<0.01$; LDHA, $\mathrm{P}<0.05)$ and in the SK-28 cells (HK, $\mathrm{P}<0.01$; M2-PK, $\mathrm{P}<0.05$; $\mathrm{PFK} 1, \mathrm{P}<0.01$; LDHA, $\mathrm{P}<0.05$ ), which was significantly increased following knockdown of CerS6. Additionally, the expression levels of glycolysis-related genes, GLUT1 and MCT1, in melanoma cells after transfection, were assessed by qPCR and western blotting, respectively. The expression of GLUT1 and MCT1 at the mRNA and protein 


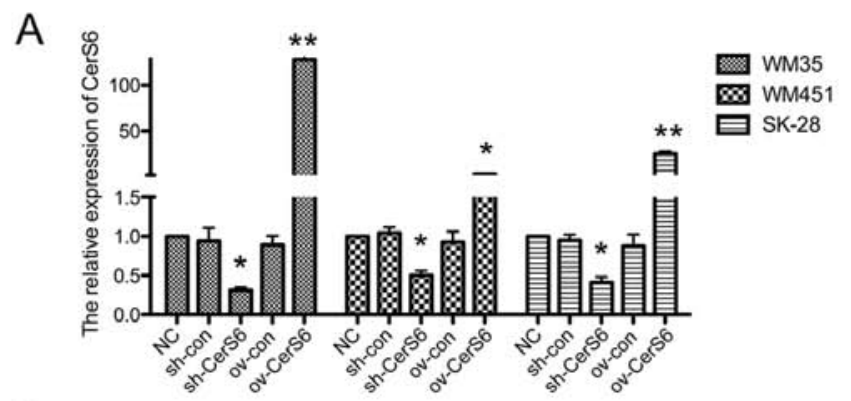

B

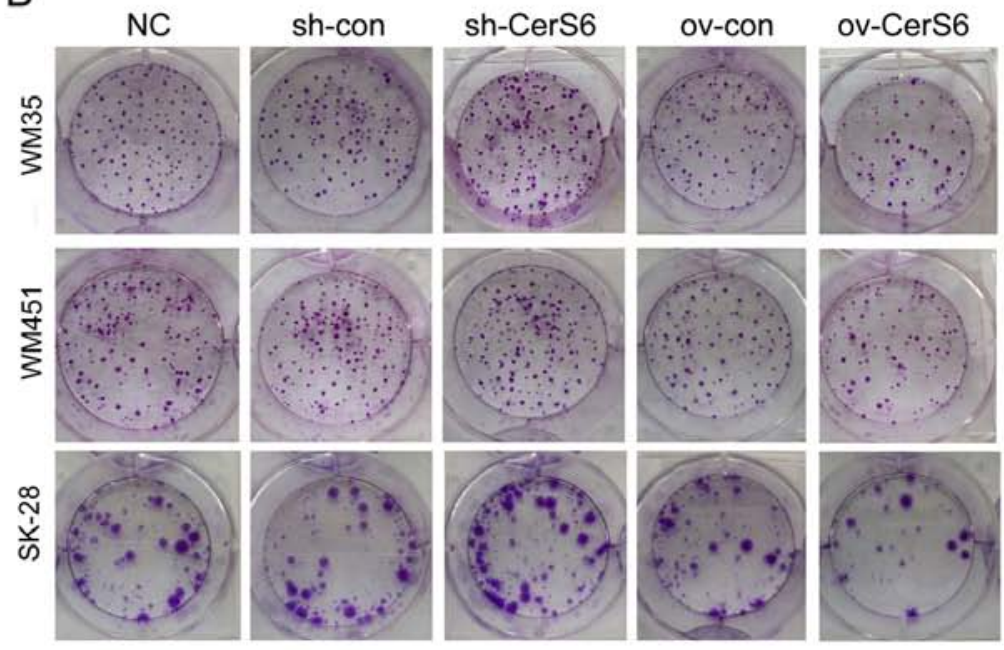

$E$
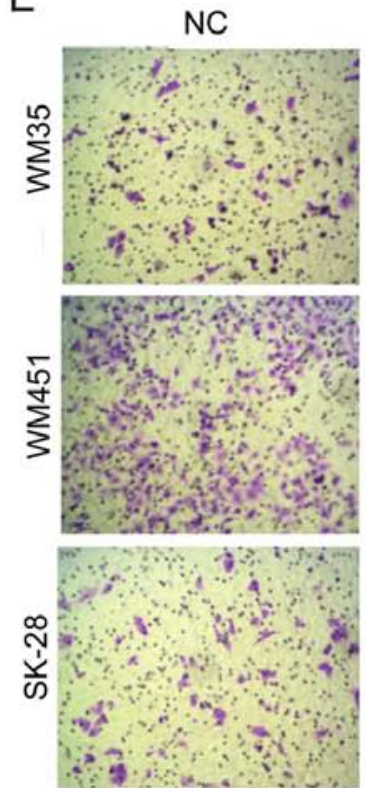

sh-con
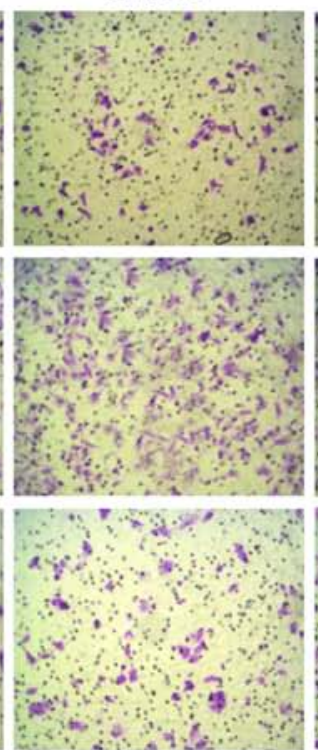

sh-CerS6
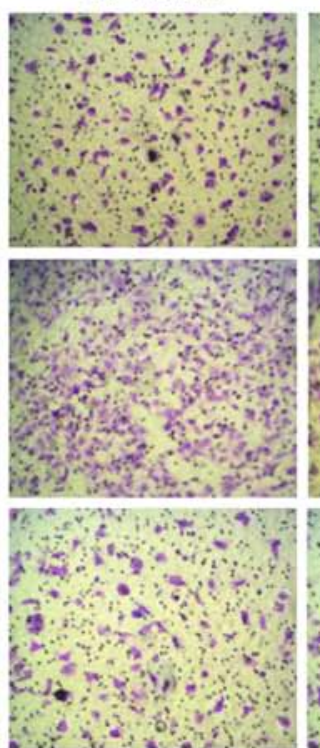

D
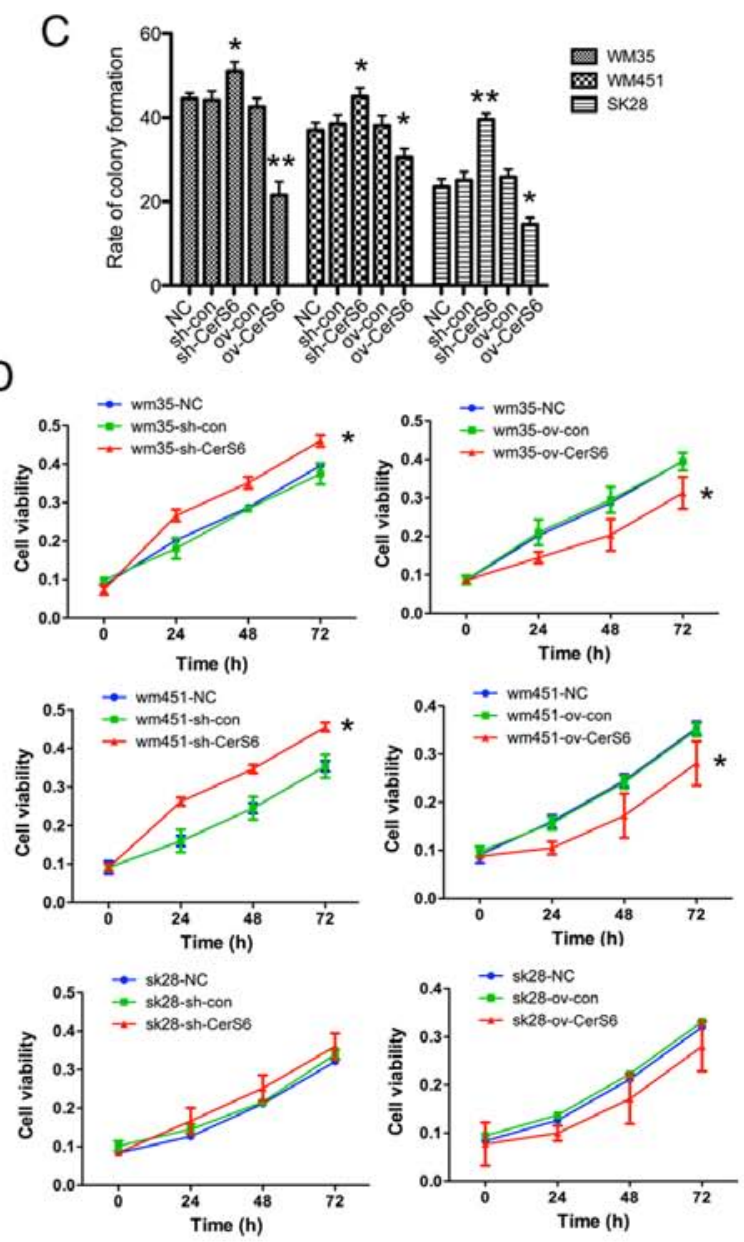

Figure 2. Silencing of CerS6 promotes the ability of invasion and proliferation in the melanoma cell lines. (A) The relative mRNA expression of CerS6 following transfection-induced overexpression (ov-CerS6) or silencing (sh-CerS6). (B) The representative images of colonies formed by melanoma cells after transfection. (C) The statistical analysis of colony formation rate in the different treated melanoma cell lines. (D) Cell viability analysis measured by MTT assay. (E) The representative images of migration as assessed by Transwell assay. Data are shown as mean $\pm \mathrm{SD}, \mathrm{n}=3$. ${ }^{*} \mathrm{P}<0.05,{ }^{* *} \mathrm{P}<0.01$.

levels was downregulated in the CerS6-overexpressing cells, and was increased after CerS6 silencing (Fig. 3B and C). Collectively, silencing of CerS6 upregulated the glycolysis in the melanoma cell lines.
Silencing of CerS6 in melanoma cell lines leads to the upregulation of GLUT1 and downregulation of WNT5A. We detected the differences in the gene expression profile between CerS6 silenced and control cells using gene chip, and found 
A
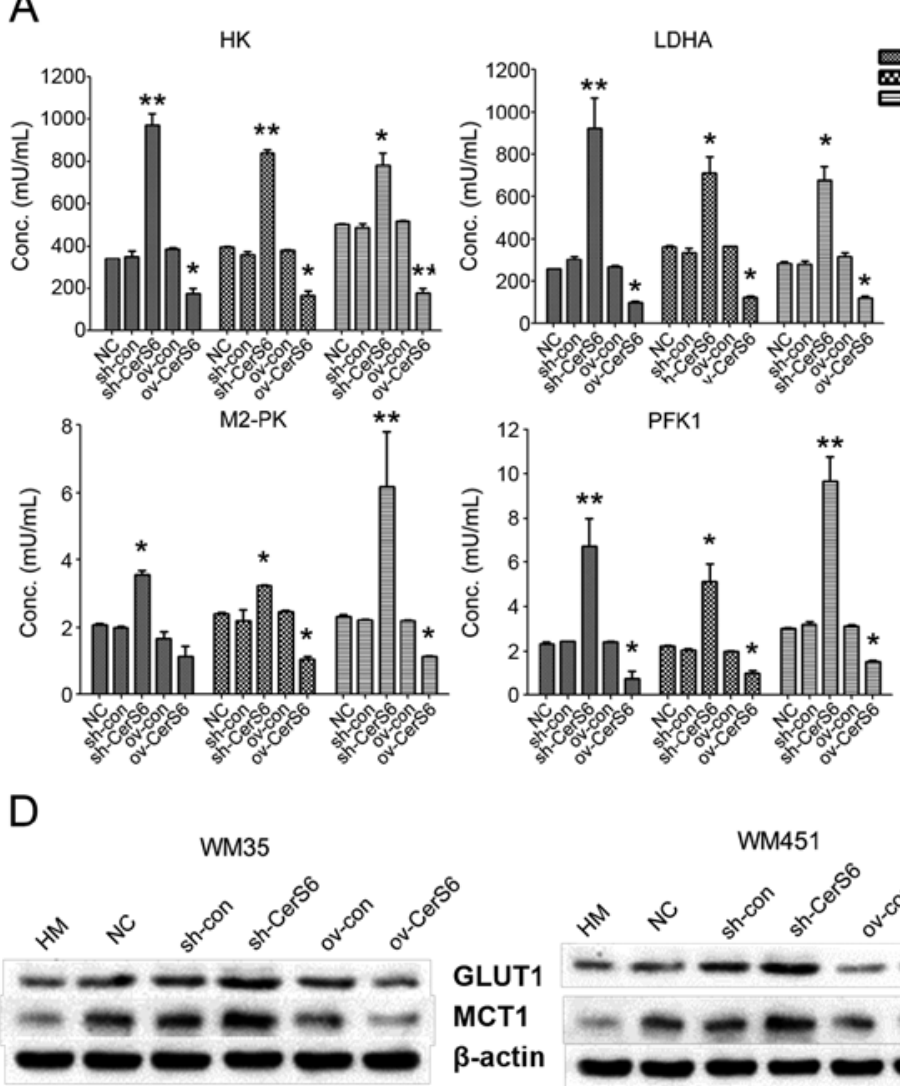

WM451

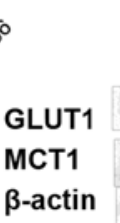

B

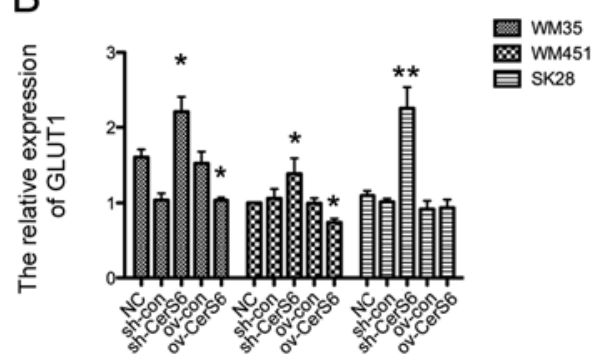

C

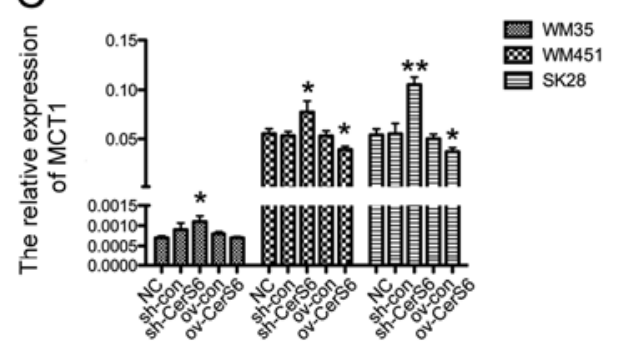

Figure 3. CerS6 upregulates glycolysis in the melanoma cells. (A) The levels of glycolysis-related enzyme activity, including HK, LDHA, M2-PK and PFK1, were measured by ELISA. (B) The relative mRNA expression of the glycolysis-related gene GLUT1 in the different treated melanoma cells. (C) The relative mRNA expression of glycolysis-related gene MCT1 in the different treated melanoma cells. (D) The representative images of GLUT1 and MCT1 protein expression in the different treated melanoma cells measured by western blotting. Data are shown as mean $\pm \mathrm{SD}, \mathrm{n}=3$. ${ }^{*} \mathrm{P}<0.05$, ${ }^{* *} \mathrm{P}<0.01$. ov-CerS6 CerS6overexpressing cells; sh-CerS6, CerS6-silenced cells.

that PDGFA, ARGHAP29, FOS, IRS1, ANGPTL4, EMP1 and BCL2L1 were upregulated in the CerS6-silenced cells. The expression of SLC2A1, which encoded the important human glucose transporter GLUT1, was also upregulated after silencing of CerS6. The downregulated genes included 8 genes, such as NKX3-1, TRAF1, NF-KB, PPAP2B, BIRC3, CXCL3, LAMC1 and WNT5A (Fig. 4A). In order to confirm the expression changes of these genes, we performed qPCR for all of these genes. As shown in Fig. 4B, no uniform change was found in three melanoma cell lines with CerS6 overexpression or silencing in 14 genes, except SLC2A1 (also known as GLUT1) and WNT5A. WNT5A was significantly downregulated in the CerS6-silenced melanoma cells, including WM35 $(\mathrm{P}<0.05)$, WM451 $(\mathrm{P}<0.05)$ and $\mathrm{SK}-28$ cell lines $(\mathrm{P}<0.05)$, and the expression of WNT5A was increased when CerS6 was overexpressed in the WM35 $(\mathrm{P}<0.001)$, WM451 $(\mathrm{P}<0.05)$ and SK-28 cell lines $(\mathrm{P}<0.05)$. Additionally, in the melanoma cell lines without any treatment, the protein expression of GLUT1 was higher compared with the Hm cells, while the expression of WNT5A was lower than that of the Hm cells (Fig. 4C). Collectively, in three CerS6-silenced melanoma cell lines, the expression of GLUT1 was upregulated while WNT5A was downregulated.

Silencing of GLUT1 promotes the expression of WNT5A and inhibits the proliferation and invasion in the melanoma cell lines. In order to confirm the role of GLUT1 in regulating the invasion and proliferation of melanoma cells, siRNA-induced silencing of GLUT1 expression was carried out. The expression of GLUT1 was significantly downregulated after siRNA transfection (Fig. 5A). WNT5A signaling was found to increase aerobic glycolysis in the melanoma cells. Accordingly, we measured the expression of WNT5A after silencing of GLUT1. Our data showed that silencing of GLUT1 led to the upregulated expression of WNT5A at the mRNA and protein level (Fig. 5B and C). Cell proliferation was measured in the WM35, WM451 and SK-28 cells by MTT assay. GLUT1-silenced cells showed statistically reduced cell viability (Fig. 5D). As shown in Fig. 5E, silencing of GLUT1 significantly decreased the number of cells that migrated through the Transwell membrane. In the colony formation assay, the number of colonies was also decreased in the GLUT1-silenced cells (Fig. 5F). These collectively indicated that silencing of GLUT1 promoted the upregulation of expression of WNT5A and inhibited the invasion and proliferation of the melanoma cell lines.

\section{Discussion}

Melanomas are prone to invasion and metastasis, and are associated with a high mortality rate (28). Accordingly, further research on the treatment of melanoma is greatly needed. The 
A

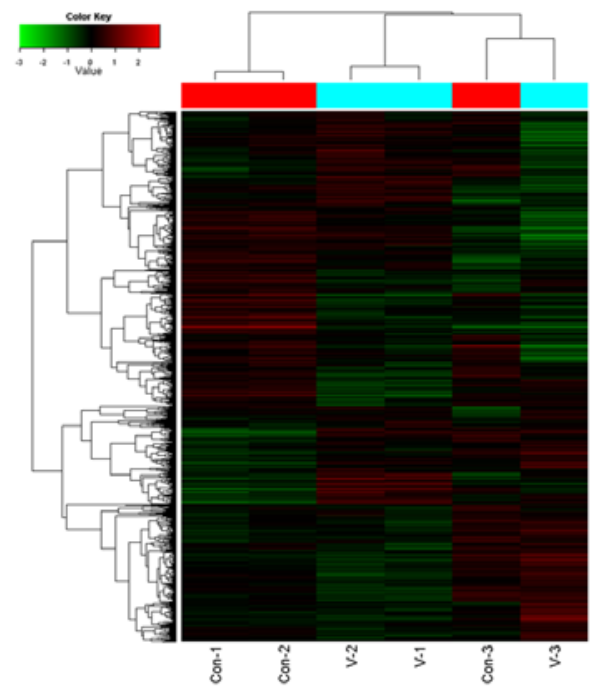

B
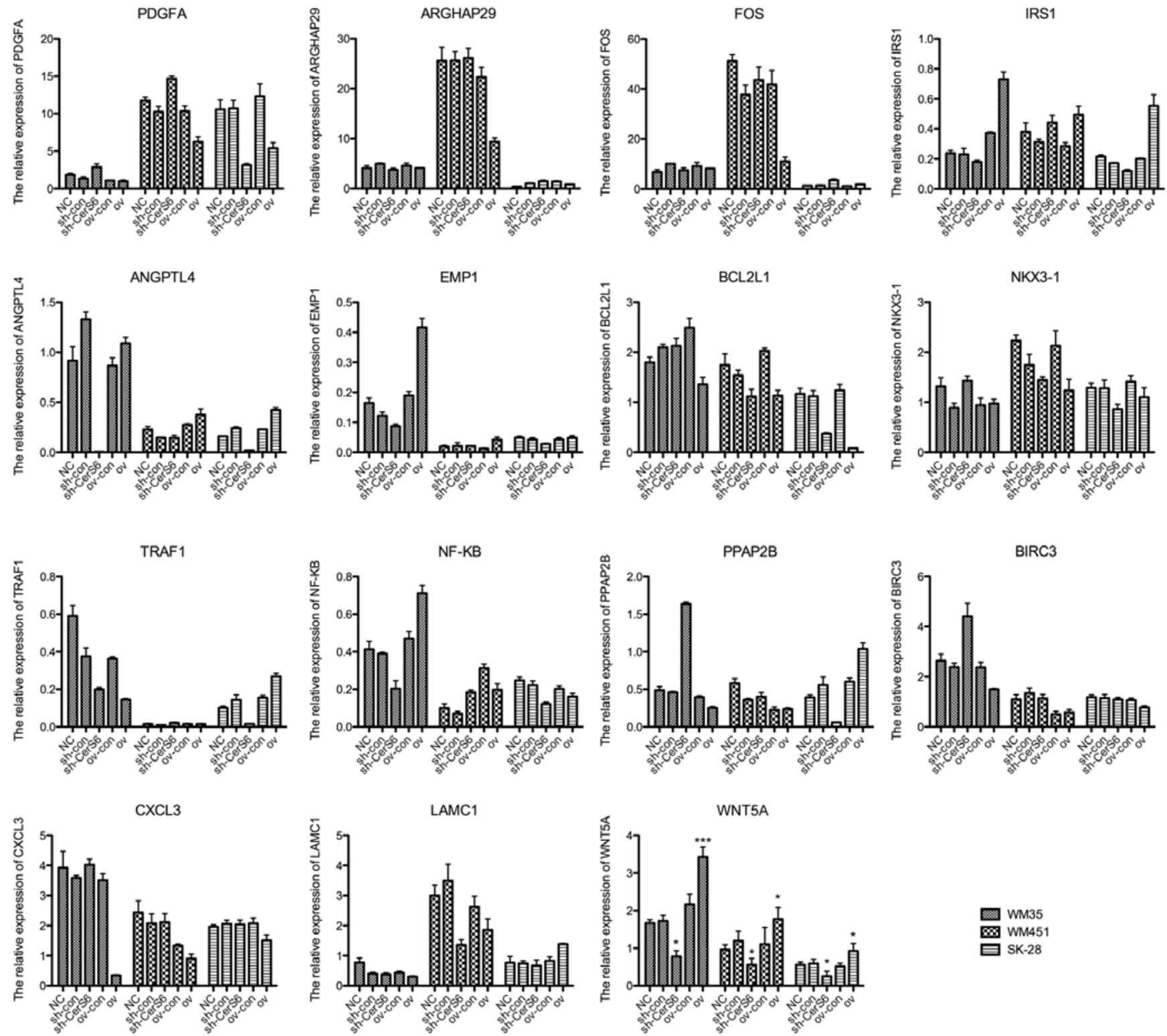

C

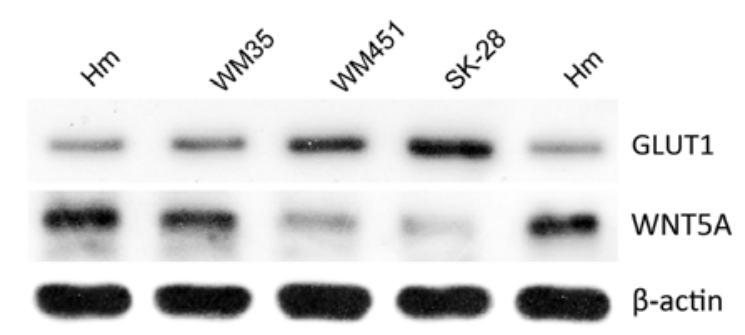

Figure 4. WNT5A is downregulated in the CerS6-silenced melanoma cell lines. (A) The representative image of gene chip detection in the CerS6-silenced melanoma cell lines, including WM35, WM451 and SK-28. (B) The relative mRNA expression of genes that were determined to be upregulated/downregulated in the gene chip. qPCR was used to confirm the expression level of these genes. (C) The representative blots of GLUT1 and WNT5A protein expression in the melanoma cell lines without any treatment. Data are shown as mean $\pm \mathrm{SD}, \mathrm{n}=3$. ${ }^{*} \mathrm{P}<0.05,{ }^{* * *} \mathrm{P}<0.001$. 
A
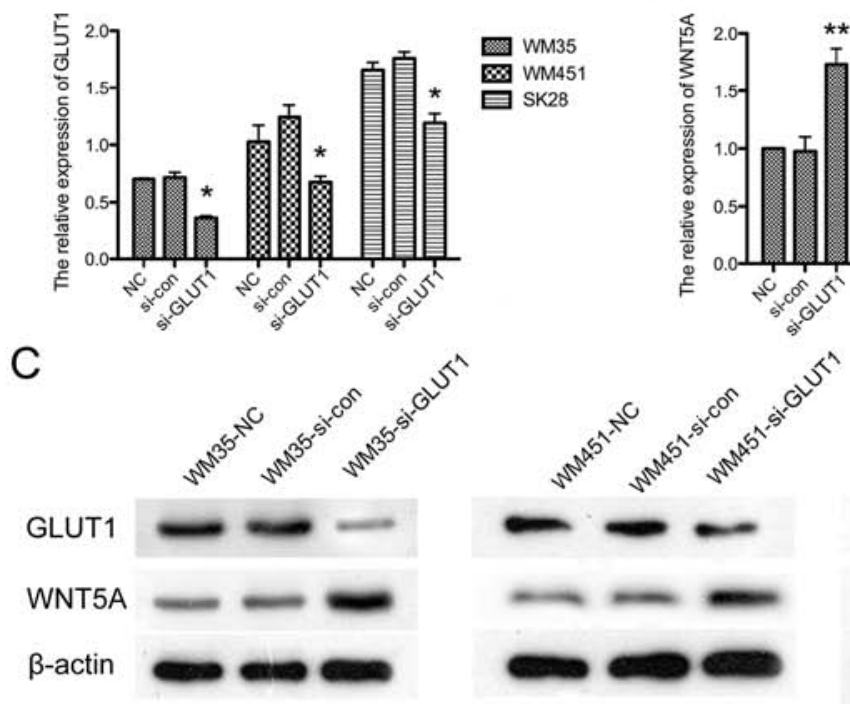

E
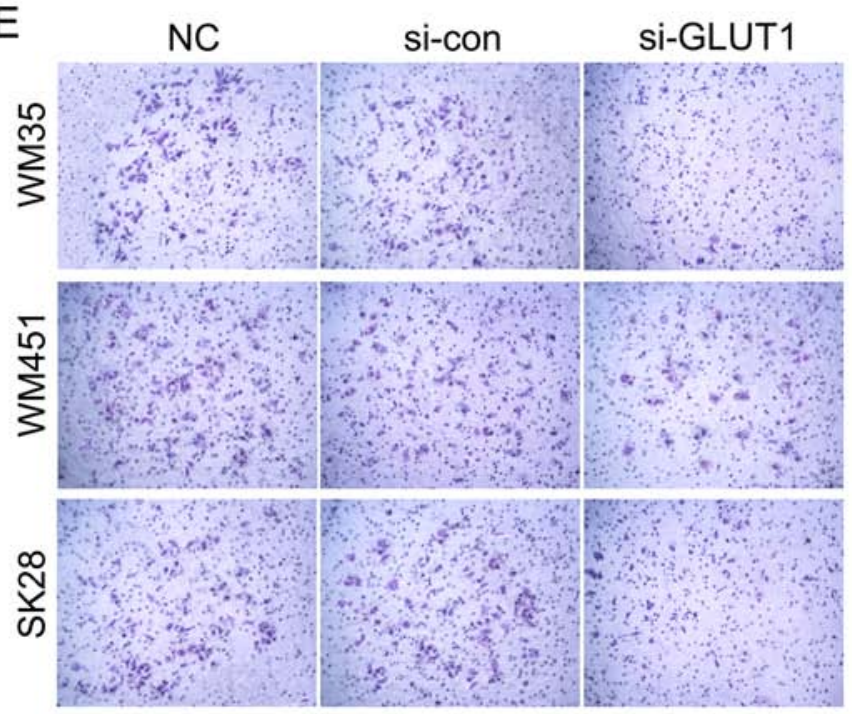

B
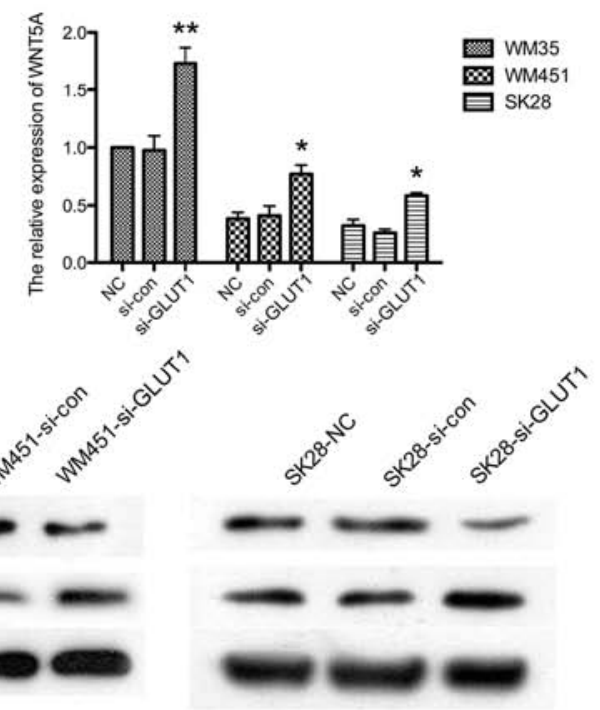

F

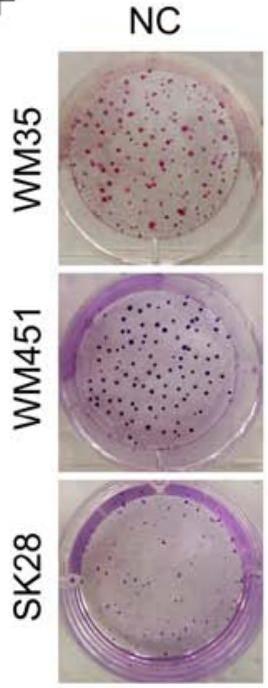

D
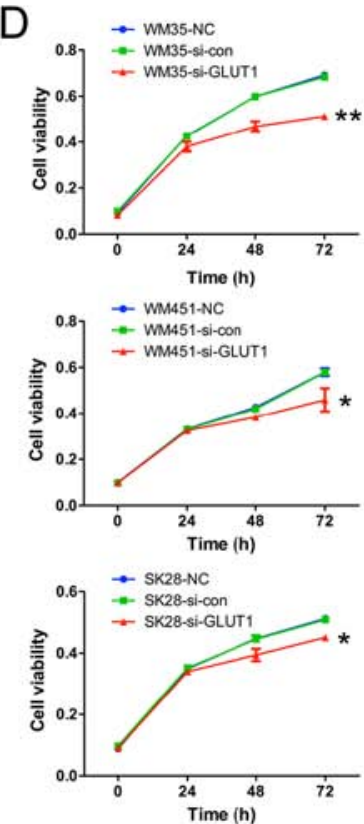

si-con

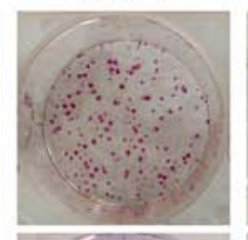

si-GLUT1

Figure 5. Silencing of GLUT1 promotes the expression of WNT5A and inhibits the proliferation and invasion of the melanoma cell lines. (A) The relative mRNA expression of GLUT1 in the siRNA-induced GLUT1-silenced cell lines. (B) The relative mRNA expression of WNT5A in the siRNA-induced GLUT1silenced cell lines. (C) The representative blots of GLUT1 and WNT5A protein expression in the siRNA-induced GLUT1-silenced cells. (D) The proliferation analysis as measured by MTT assay. (E) The representative images of migration from the Transwell assay. (F) The representative images of colonies formed by cells following the different treatments. Data are shown as mean $\pm \mathrm{SD}, \mathrm{n}=3 .{ }^{*} \mathrm{P}<0.05,{ }^{* *} \mathrm{P}<0.01$.

'Warburg effect' states that cancer cells take up increased glucose compared to their non-transformed counterparts and preferentially utilize glycolytic metabolism to produce ATP, even in the presence of oxygen, a phenomenon termed aerobic glycolysis (5). Accordingly, glycolysis is a potential target of cancer therapy. CerSs, which consists of six members, CerS1 to CerS6, regulates sphingolipid synthesis and numerous aspects of cell biology, including cell growth, apoptosis, autophagy and particularly cancer development (18-21). In the present study, we analyzed the effect and mechanism of CerS6 in the regulation of the invasion and glycolysis of melanoma. CerS6 was found to be lowly expressed in the melanoma cell lines when compared with the normal human epithelial cells. Silencing of CerS6 promoted the invasion and proliferation of the melanoma cell lines. Additionally, CerS6 upregulated the activity of glycolysis-related enzymes and the expression of glycolysis-related genes including GLUT1 and MCT1. Furthermore, using gene chip and qPCR confirmation, we found the expression of glycolysis-related gene SLC2A1 which encoded protein GLUT1 was upregulated in the CerS6-silenced melanoma cells, and notably WNT5A was downregulated. Silencing of GLUT1 resulted in the increased expression of WNT5A in the melanoma cells, and inhibited the invasion and proliferation capability of the melanoma cells.

Recent data revealed that CerSs play important roles in cancer regulation. CerS1-dependent generation of C18-ceramide plays important roles in the pathogenesis of head and neck squamous cell carcinoma (HNSCC) $(29,30)$. Additionally, as an important member of the CerS family, various studies also suggest that CerS6 is involved in cancer etiology. Knockdown of CerS6 resulted in the decreased expression of C16-ceramide, and reduced TRAIL-induced 
apoptosis in colon adenocarcinoma cells, while upregulated expression of CerS6 sensitized the cells to TRAIL (22). Moreover, downregulation of CerS6 in HNSCC induced ER stress, and increased HNSCC tumor development and growth were observed in CerS6-overexpressing cells (24). Furthermore, a microarray study indicated CerS6 as an important participant in cancer differentiation and early embryonic development (31). In the present study, we found that CerS6 regulated the ability of proliferation, invasion and glycolysis in the melanoma cell lines. Its suppressed expression in melanoma cells may play essential roles in the development of melanoma, and further research on targeting CerS6 for melanoma treatment is warranted.

The role of CerS6 in cell apoptosis is distinctively reported in different tissues. Senkal et al showed the anti-apoptotic role of CerS6 in HNSCC (23). However, developmentally regulated CerS6 was found to increase mitochondrial $\mathrm{Ca}^{2+}$ loading capacity and promote apoptosis (32). Similarly, CerS6 knockdown inhibited TRAIL-induced apoptosis (22). In the present study, we detected the cell viability and colony formation capability of melanoma cell lines after CerS6 overexpression and silencing. We found that the decreased expression of CerS6 in melanoma cell lines led to the promotion of cell viability and colony formation capability.

After silencing of CerS6, we found that the activity of glycolysis-related enzymes was upregulated, as well as the expression of glycolysis-related genes GLUT1 and MCT1. The upregulated expression of GLUT1 decreased the expression of WNT5A, the signaling of which in malignant melanoma cells alters cellular energy metabolism and specifically increases aerobic glycolysis (33). In a recent study, WNT5A was suggested to promote melanoma cell migration/invasion via an FZD-4- $\beta$-catenin-dependent mechanism (34). This is not in accordance with our findings. In the present study, the upregulation of WNT5A induced by the silencing of GLUT1 was accompanied by decreased invasion capability in the melanoma cells. The reason for the descrepant results between our results and the previous study may be associated with different signaling pathways, or the downregulated invasion in our study may not be directly regulated by WNT5A.

There is a limitation to our study. We investigated the role of CerS6 which relied on overexpression and silencing of single CerS6 protein as a gain-of-function model. However, little is known about the regulation and interregulation of other CerS proteins with regard to maintaining overall sphingolipid homeostasis. Mullen et al demonstrated that the ability of CerS knockdown to cause increases in sphingolipids was exemplified by their attempted simultaneous knockdown of CerS2/5/6 (35). Unlike individual knockdown of CerS2 and CerS6, the resulting increases in sphingolipids imply that there is a significant response of cells to the reduced CerS expression or activity that involves the accumulation of these lipids. Accordingly, the regulatory role of a single CerS still needs further confirmation in a more systemic circumstance.

Collectively, CerS6 promoted the invasion and glycolysis in melanoma cells. This provides a new mechanism of CerS6 action in melanoma cells and should further establish its future use as a valid chemopreventive and chemotherapeutic agent against melanoma.

\section{Acknowledgements}

The present study was supported by the National Natural Science Foundation of China (nos. 81572689, 81372140, 81301688, 81272192 and 81171882), the Ph.D. Programs Foundation of the Ministry of Education of China (nos. 20130162110050 and 20130162120093), the Natural Science Foundation of Hunan Province (no. 2015JJ4053), the Project of Science and Technology of Hunan Province (nos. 2014SK2018 and 2015SF2066-2), the Program for New Century Excellent Talents in University (NCET-11-0527), the Postdoctoral Foundation of Central South University (no. 131425), and the '125 Talent Project' and 'New Xiangya Talent Project' of the Third Xiangya Hospital of Central South University.

\section{References}

1. Xu D, Tan J, Zhou M, Jiang B, Xie H, Nie X, Xia K and Zhou J: Let-7b and microRNA-199a inhibit the proliferation of B16F10 melanoma cells. Oncol Lett 4: 941-946, 2012.

2. Thompson JF, Scolyer RA and Kefford RF: Cutaneous melanoma. Lancet 365: 687-701, 2005.

3. Hanahan D and Weinberg RA: Hallmarks of cancer: The next generation. Cell 144: 646-674, 2011.

4. Stathem M, Marimuthu S, O'Neal J, Rathmell JC, Chesney JA, Beverly LJ and Siskind LJ: Glucose availability and glycolytic metabolism dictate glycosphingolipid levels. J Cell Biochem 116: 67-80, 2015.

5. Vander Heiden MG, Cantley LC and Thompson CB: Understanding the Warburg effect: The metabolic requirements of cell proliferation. Science 324: 1029-1033, 2009.

6. Warburg O: On the origin of cancer cells. Science 123: 309-314, 1956.

7. Hannun YA and Obeid LM: Principles of bioactive lipid signalling: Lessons from sphingolipids. Nat Rev Mol Cell Biol 9: 139-150, 2008.

8. Ogretmen B: Sphingolipids in cancer: Regulation of pathogenesis and therapy. FEBS Lett 580: 5467-5476, 2006.

9. Ogretmen B and Hannun YA: Biologically active sphingolipids in cancer pathogenesis and treatment. Nat Rev Cancer 4: 604-616, 2004.

10. Mathias S, Peña LA and Kolesnick RN: Signal transduction of stress via ceramide. Biochem J 335: 465-480, 1998.

11. Knapp P, Baranowski M, Knapp M, Zabielski P, BłachnioZabielska AU and Górski J: Altered sphingolipid metabolism in human endometrial cancer. Prostaglandins Other Lipid Mediat 92: 62-66, 2010.

12. Wu J, Cheng Y, Nilsson A and Duan RD: Identification of one exon deletion of intestinal alkaline sphingomyelinase in colon cancer HT-29 cells and a differentiation-related expression of the wild-type enzyme in Caco-2 cells. Carcinogenesis 25: 1327-1333, 2004.

13. Bektas M, Jolly PS, Müller C, Eberle J, Spiegel S and Geilen CC: Sphingosine kinase activity counteracts ceramide-mediated cell death in human melanoma cells: Role of Bcl-2 expression. Oncogene 24: 178-187, 2005.

14. Samsel L, Zaidel G, Drumgoole HM, Jelovac D, Drachenberg C, Rhee JG, Brodie AM, Bielawska A and Smyth MJ: The ceramide analog, B13, induces apoptosis in prostate cancer cell lines and inhibits tumor growth in prostate cancer xenografts. Prostate 58: 382-393, 2004.

15. Gouazé V, Liu YY, Prickett CS, Yu JY, Giuliano AE and Cabot MC: Glucosylceramide synthase blockade down-regulates P-glycoprotein and resensitizes multidrug-resistant breast cancer cells to anticancer drugs. Cancer Res 65: 3861-3867, 2005.

16. Levy $M$ and Futerman AH: Mammalian ceramide synthases. IUBMB Life 62: 347-356, 2010.

17. Pewzner-Jung Y, Ben-Dor S and Futerman AH: When do Lasses (longevity assurance genes) become CerS (ceramide synthases)?: Insights into the regulation of ceramide synthesis. J Biol Chem 281: 25001-25005, 2006.

18. Teufel A, Maass T, Galle PR and Malik N: The longevity assurance homologue of yeast lag1 (Lass) gene family (Review). Int J Mol Med 23: 135-140, 2009. 
19. Harel R and Futerman AH: Inhibition of sphingolipid synthesis affects axonal outgrowth in cultured hippocampal neurons. J Biol Chem 268: 14476-14481, 1993.

20. Bose R, Verheij M, Haimovitz-Friedman A, Scotto K, Fuks Z and Kolesnick R: Ceramide synthase mediates daunorubicininduced apoptosis: An alternative mechanism for generating death signals. Cell 82: 405-414, 1995.

21. Spassieva SD, Mullen TD, Townsend DM and Obeid LM Disruption of ceramide synthesis by CerS2 down-regulation leads to autophagy and the unfolded protein response. Biochem J 424: 273-283, 2009

22. White-Gilbertson S, Mullen T, Senkal C, Lu P, Ogretmen B, Obeid L and Voelkel-Johnson C: Ceramide synthase 6 modulates TRAIL sensitivity and nuclear translocation of active caspase-3 in colon cancer cells. Oncogene 28: 1132-1141, 2009.

23. Senkal CE, Ponnusamy S, Rossi MJ, Bialewski J, Sinha D, Jiang JC, Jazwinski SM, Hannun YA and Ogretmen B: Role of human longevity assurance gene 1 and $\mathrm{C}_{18}$-ceramide in chemotherapy-induced cell death in human head and neck squamous cell carcinomas. Mol Cancer Ther 6: 712-722, 2007.

24. Senkal CE, Ponnusamy S, Bielawski J, Hannun YA and Ogretmen B: Antiapoptotic roles of ceramide-synthase-6-generated $\mathrm{C}_{16}$-ceramide via selective regulation of the $\mathrm{ATF} 6 / \mathrm{CHOP}$ arm of ER-stress-response pathways. FASEB J 24: 296-308, 2010.

25. Zhou J, Liu R, Wang Y, Tang J, Tang S, Chen X, Xia K, Xiong W, $\mathrm{Xu}$ D, Wang S, et al: miR-199a-5p regulates the expression of metastasis-associated genes in B16F10 melanoma cells. Int J Clin Exp Pathol 7: 7182-7190, 2014

26. Zhou J, Xu D, Xie H, Tang J, Liu R, Li J, Wang S, Chen X, Su J, Zhou X, Xia K, He Q, Chen J, Xiong W, Cao P, Cao K: miR-33a functions as a tumor suppressor in melanoma by targeting HIF-1 $\alpha$. Cancer Biol Ther 16: 846-855, 2015

27. Zhang Y, Liu H, Jin J, Zhu X, Lu L, Jiang H: The role of endogenous reactive oxygen species in oxymatrine-induced caspase-3-dependent apoptosis in human melanoma A375 cells. Anticancer Drugs 21: 494-501, 2010.

28. Sun J, Han J, Zhu Q, Li Z, Hu J: Camptothecin fails to induce apoptosis in tumor necrosis factor-alpha-treated HaCaT cells. Pharmacology 89: 58-63, 2012.
29. Koybasi S, Senkal CE, Sundararaj K, Spassieva S, Bielawski J, Osta W, Day TA, Jiang JC, Jazwinski SM, Hannun YA, et al: Defects in cell growth regulation by $\mathrm{C}_{18 \cdot 0}$-ceramide and longevity assurance gene 1 in human head and neck squamous cell carcinomas. J Biol Chem 279: 44311-44319, 2004.

30. Karahatay S, Thomas K, Koybasi S, Senkal CE, Elojeimy S, Liu X, Bielawski J, Day TA, Gillespie MB, Sinha D, et al: Clinical relevance of ceramide metabolism in the pathogenesis of human head and neck squamous cell carcinoma (HNSCC): Attenuation of $\mathrm{C}_{18}$-ceramide in HNSCC tumors correlates with lymphovascular invasion and nodal metastasis. Cancer Lett 256 : 101-111, 2007.

31. Weinmann A, Galle PR and Teufel A: LASS6, an additional member of the longevity assurance gene family. Int $\mathbf{J}$ Mol Med 16: 905-910, 2005.

32. Novgorodov SA, Chudakova DA, Wheeler BW, Bielawski J, Kindy MS, Obeid LM and Gudz TI: Developmentally regulated ceramide synthase 6 increases mitochondrial $\mathrm{Ca}^{2+}$ loading capacity and promotes apoptosis. J Biol Chem 286: 4644-4658, 2011.

33. Sherwood V, Chaurasiya SK, Ekström EJ, Guilmain W, Liu Q, Koeck T, Brown K, Hansson K, Agnarsdóttir M, Bergqvist M, et al: WNT5A-mediated $\beta$-catenin-independent signalling is a novel regulator of cancer cell metabolism. Carcinogenesis 35: 784-794, 2014.

34. Grossmann AH, Yoo JH, Clancy J, Sorensen LK, Sedgwick A Tong Z, Ostanin K, Rogers A, Grossmann KF, Tripp SR, et al: The small GTPase ARF6 stimulates $\beta$-catenin transcriptional activity during WNT5A-mediated melanoma invasion and metastasis. Sci Signal 6: ra14, 2013.

35. Mullen TD, Spassieva S, Jenkins RW, Kitatani K, Bielawski J, Hannun YA and Obeid LM: Selective knockdown of ceramide synthases reveals complex interregulation of sphingolipid metabolism. J Lipid Res 52: 68-77, 2011. 